\title{
Complete reversal of acid-induced acute lung injury by blocking of platelet-neutrophil aggregation
}

\author{
Alexander Zarbock,, ${ }^{1,2}$ Kai Singbartl,2 and Klaus Ley 1,3,4 \\ ${ }^{1}$ Robert M. Berne Cardiovascular Research Center, University of Virginia, Charlottesville, Virginia, USA. \\ 2Department of Anesthesiology and Intensive Care Medicine, University of Münster, Münster, Germany. ${ }^{3}$ Department of Physiology and \\ Biological Physics and ${ }^{4}$ Department of Biomedical Engineering, University of Virginia, Charlottesville, Virginia, USA.
}

\begin{abstract}
Acute lung injury (ALI) causes high mortality, but its molecular mechanisms are poorly understood. Acid aspiration is a frequent cause of ALI, leading to neutrophil sequestration, increased permeability, and deterioration of gas exchange. We investigated the role of platelet-neutrophil interactions in a murine model of acid-induced ALI. Acid aspiration induced P-selectin-dependent platelet-neutrophil interactions in blood and in lung capillaries. Reducing circulating platelets or blocking P-selectin halted the development of ALI. Bone marrow chimeras showed that platelet, not endothelial, P-selectin was responsible for the injury. The interaction of platelets with neutrophils and endothelia was associated with $\mathrm{TXA}_{2}$ formation, with detrimental effects on permeability and tissue function. Activated platelets induced endothelial expression of ICAM-1 and increased neutrophil adhesion. Inhibition of platelet-neutrophil aggregation improved gas exchange, reduced neutrophil recruitment and permeability, and prolonged survival. The key findings were confirmed in a sepsis-induced model of ALI. These findings may translate into improved clinical treatments for ALI.
\end{abstract}

\section{Introduction}

Acute lung injury (ALI) is a common disease with an incidence of 79 per 100,000 person-years in the United States (1). Despite the use of state-of-the-art treatment, this disease is associated with high mortality of up to $38 \%$ (1). Sepsis, trauma, and massive transfusion are all extrapulmonary causes of ALI whereas pneumonia and acid aspiration are typical causes of intrapulmonary ALI. Aspiration is a regular complication of general anesthesia $(2,3)$ and can also occur in critically ill patients and unconscious patients (4). Acid aspiration in WT mice, which mimics human ALI (5-7), can be induced experimentally by inflicting $\mathrm{HCl}$-induced chemical damage of the alveolar-capillary membrane (8), resulting in polymorphonuclear neutrophil (PMN) recruitment (9), pulmonary edema, and impairment of gas exchange (7).

Recruitment of PMNs into the lung is a key event in the development of ALI (10). PMN activation, sequestration, and emigration into the lung proceed in a sequence of overlapping events (11). In contrast with the well-characterized multistep adhesion cascade of leukocyte recruitment in systemic microcirculation, the molecular requirements of leukocyte recruitment into the lung are incompletely defined. Due to the anatomic and molecular properties of the lung, PMN recruitment is only partially $\beta_{2}$ integrin dependent (12).

Neutrophils can interact with adherent platelets and leukocytes in a process called secondary capture, which is often followed by neutrophil-endothelial interactions $(13,14)$. Platelet glycoprotein $\mathrm{Ib} \alpha(\mathrm{GPIb} \alpha)(15)$ and GPIIb/GPIIIa (CD41/CD61, $\alpha_{\mathrm{II}} \beta_{3}$ integrin)

Nonstandard abbreviations used: ALI, acute lung injury; ASS, acetylsalicylic acid; BAL, bronchoalveolar lavage; GP, glycoprotein; HPMEC, human pulmonary microvascular endothelial cell; PMN, polymorphonuclear neutrophil; Selp $p^{-/-}$, selectin $\mathrm{P}^{-/-}$(mice); $\mathrm{TP}$, thromboxane receptor.

Conflict of interest: The authors have declared that no conflict of interest exists. Citation for this article: J. Clin. Invest. 116:3211-3219 (2006). doi:10.1172/JCI29499.
(16), endothelial P-selectin (17), and von Willebrand factor (18) have been shown to play important roles in platelet-endothelial interactions. Platelet depletion leads to diminished leukocyte recruitment in many models $(19,20)$. Neutrophil-platelet interactions are mainly mediated by P-selectin GP ligand 1 (PSGL-1), which is expressed on neutrophils and which binds to platelet P-selectin $(21,22)$. Firm adhesion of PMNs to platelets is mediated by the $\beta_{2}$-integrin macrophage antigen-1 (Mac-1, also known as $\mathrm{CD} 11 \mathrm{~b} / \mathrm{CD} 18$ ), which binds platelet GPIb $\alpha$, and the simultaneous binding of fibrinogen to platelet GPIIb/GPIIIa and CD11b/ CD18 on PMNs. These interactions lead to PMN activation by a process of outside-in signaling through integrins $(23,24)$ in addition to the presentation of chemokines and lipid mediators by platelets to PMNs (25-27).

Platelets can be activated by thrombin, ADP, platelet-activating factor, and $\mathrm{TXA}_{2}(28)$. TXA 2 is an arachidonic acid metabolite that is generated in several cell types and tissues, such as platelets, inflammatory cells, and pulmonary tissue, by the enzymes cyclooxygenase, hydroperoxidase, and tissue-specific isomerases $(29,30)$. The binding of $\mathrm{TXA}_{2}$ to the $\mathrm{G}$ protein-coupled thromboxane receptors (TPs) leads to a broad range of cellular responses, including integrin activation, platelet aggregation, contraction of smooth muscle cells, and increased vascular permeability, and can be involved in the development of ALI (31). Platelets express TP $\alpha$, and endothelial cells express both TP $\alpha$ and TP $\beta$ (29). Stimulation of endothelial TPs induces an activation of $\mathrm{G}$ protein-dependent pathways, leading to recruitment of numerous downstream effector targets that increase the surface expression of adhesion molecules such as ICAM-1 in a PKC-dependent manner (29).

In the present study, we investigated the role of platelet-neutrophil interactions and $\mathrm{TXA}_{2}$ production in murine models of $\mathrm{HCl}$ - or sepsis-induced ALI and in vitro in a human pulmonary endothelial cell culture system. In order to investigate the mol- 

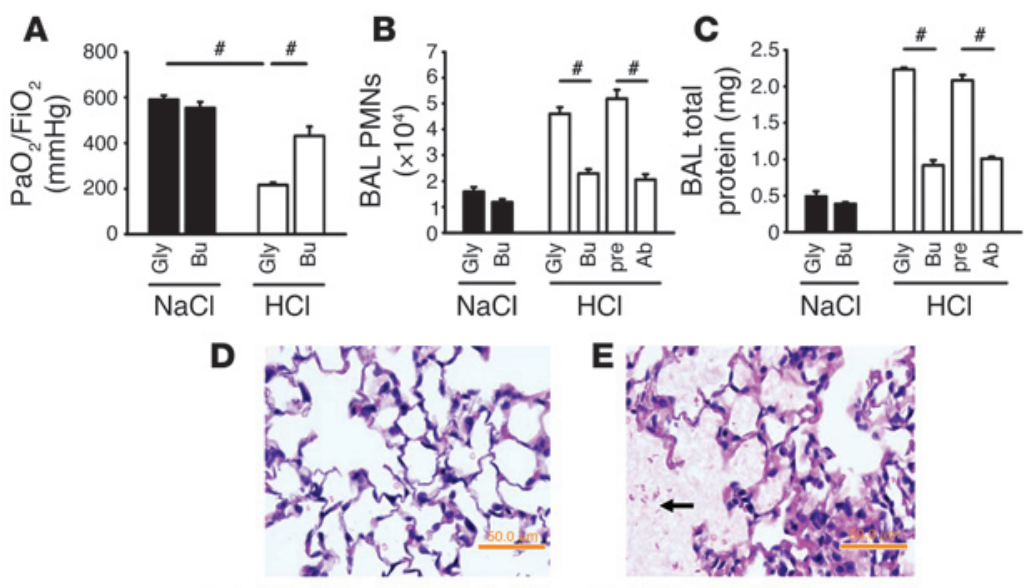

$\mathbf{F}$
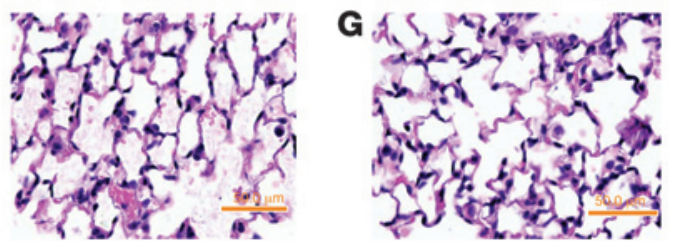

\section{Figure 1}

Platelets control PMN recruitment into the lung in acidinduced ALI. (A-C) Platelet depletion (by $40 \%$ ) prior to acid application by busulfan ( $n=4-10$ mice per group) significantly improved gas exchange (A), reduced intravascular and interstitial PMN accumulation (data not shown), diminished PMNs in the BAL fluid (B), and partially prevented increased vascular permeability (C). Platelet depletion ( $85 \%$ ) caused by a polyclonal $\mathrm{Ab}$ diminished PMN recruitment into the alveolar space (B) and permeability (C). (D-G) Photomicrographs of lung tissue. H\&E-stained paraffin sections from control mice (D) and mice 2 hours after acid administration (E). Acid application induced increased permeability with an influx of protein-rich fluid and cells (E, arrow) into the alveolar space, swelling of the interstitium, and cell accumulation in the interstitial space (E). Glycol pretreatment $(\mathbf{F})$ prior to initiation of ALI induced the same histological changes seen in untreated mice after acid application whereas the pretreatment with busulfan led to reduced morphological changes (G). Original magnification, $\times 65$. Gly, glycol; $\mathrm{Bu}$, busulfan; pre, preimmune serum; $\mathrm{Ab}$, polyclonal antiplatelet Abs. ${ }^{P} P<0.05$. Scale bars, $50.0 \mu \mathrm{m}$. ecules responsible for platelet-neutrophil interactions, chimeric mice were used that expressed P-selectin on hematopoietic cells, nonhematopoietic cells, both, or neither. The role of $\mathrm{TXA}_{2}$ was investigated by a specific receptor antagonist. Our in vivo and in vitro data show that platelet-neutrophil interactions promote the formation of $\mathrm{TXA}_{2}$ and are major contributors to acid-induced PMN recruitment and lung damage. Blocking P-selectin-dependent platelet-neutrophil interactions was highly protective in this model of acid-induced ALI.

\section{Results}

Platelets control neutrophil recruitment into the lung. In order to investigate the role of platelets in ALI, we depleted platelets by injecting mice with busulfan, which reduced blood platelet counts by $40 \%$ without affecting other blood cells (Supplemental Table 1; supplemental material available online with this article; doi:10.1172/JCI29499DS1). Platelet depletion significantly $(P<0.05)$ prevented the deterioration of gas exchange following $\mathrm{HCl}$ application (Figure $1 \mathrm{~A}$ ). To investigate the $\mathrm{PMN}$ recruitment pattern, including intravascular accumulation, transendothelial migration, and transepithelial migration, PMNs in lung homogenates were identified by flow cytometry (11). Two hours after acid instillation, HCl-treated mice showed a significant accumulation of PMNs in the intravascular and interstitial compartments compared with control mice (data not shown). Platelet depletion prior to the induction of ALI diminished the accumulation of PMNs in the intravascular, interstitial (data not shown), and alveolar compartments (Figure 1B). Furthermore, the depletion of platelets reduced protein leakage as determined by bronchoalveolar lavage (BAL) fluid protein levels (Figure 1C). Both findings were confirmed by depleting platelets with an antiplatelet $\mathrm{Ab}$, which reduced platelet counts by $85 \%$ without affecting leukocytes. Histological analysis of the lungs following $\mathrm{HCl}$ application revealed alveolar septal thickening, accumulation of cells within the interstitial compartment, and influx of protein-rich fluid into the alveolar space (Figure 1E) compared with control mice (Figure 1D). Platelet depletion by busulfan prior to the induction of ALI led to a reduction of histological changes (Figure 1G) compared with the control group (Figure 1F).

Acid aspiration-induced ALI induces platelet-neutrophil interaction. Flow cytometric analysis of whole ventricular blood revealed the presence of platelet-neutrophil aggregates 30 minutes after acid application, as detected by a significant increase in the percentage of PMNs $\left(\mathrm{CD}_{4} 5^{+}, \mathrm{Gr}-1^{+}, 7 / 4^{+}\right)$positive for the platelet-specific marker CD41 (Figure 2, A and B). Depletion of platelets by Abs led to a significant reduction of platelet-neutrophil aggregates in the blood 30 minutes after induction of ALI (Figure 2C). The application of a P-selectin Ab 15 minutes after induction of ALI diminished platelet-neutrophil aggregates in the blood to baseline levels, suggesting that platelet-neutrophil aggregate formation is P-selectin dependent (Figure 2B). Flow cytometry data were confirmed by transmission electron microscopy showing numerous platelet-neutrophil aggregates in pulmonary microvascular vessels 30 minutes after initiation of acid-induced ALI (Figure 2D).

Hematopoietic P-selectin mediates the development of ALI. The increase in P-selectin-dependent platelet-neutrophil aggregates upon intratracheal acid application suggested that P-selectin blockade may lead to improvement of functional and morphological parameters. Mice treated with a P-selectin Ab 15 minutes after induction of ALI displayed a significant improvement in the oxygenation index $(P<0.05)$ compared with the control mice (Figure 3A). P-selectin blockade reduced $\mathrm{PMN}$ migration into the interstitial (data not shown) and alveolar compartments (Figure 3B) as well as protein leakage into the BAL fluid (Figure 3C). In accordance with these findings, the histological analysis of the lung after P-selectin blockade displayed reduced pathological changes (Supplemental Figure 1A).

To determine whether platelet or endothelial P-selectin was responsible for PMN recruitment and the development of ALI, we generated BM chimeric mice. Consistent with the findings in mice treated with P-selectin Abs, mice lacking platelet and endothelial P-selectin were protected from ALI. This protection was accompanied by an improvement of gas exchange (Figure 3D) and a reduc- 
A
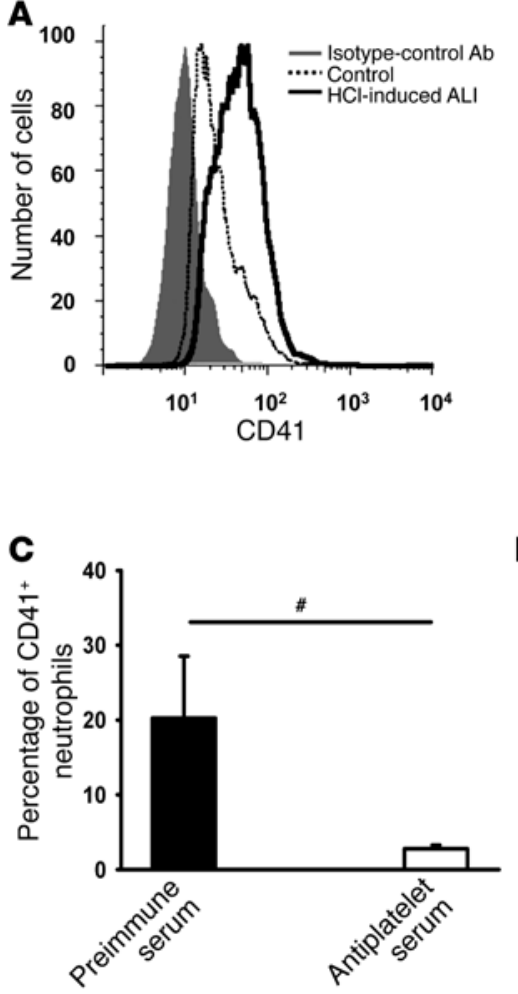

B

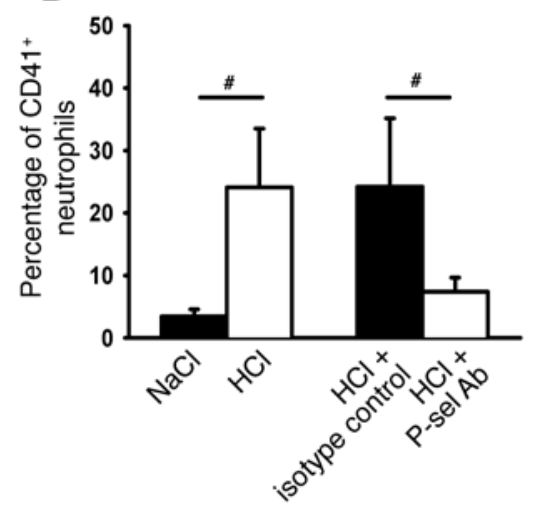

D

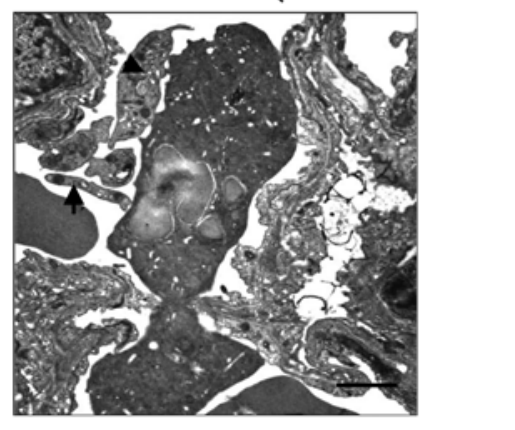

Figure 2

Acid-induced ALI causes platelet-neutrophil interactions. (A) Flow cytometry analysis of platelet-neutrophil aggregates after initiation of $\mathrm{HCl}$-induced $\mathrm{ALI}$. CD45, Gr-1, and 7/4 mAbs were used to identify PMNs (data not shown). Neutrophil-platelet aggregates were identified as neutrophils that were also positive for the platelet-specific marker CD41. (B) Thirty minutes after initiation of $\mathrm{HCl}$-induced $\mathrm{ALI}$, platelet-neutrophil interaction in the blood increased significantly. P-selectin Abs almost completely prevented the formation of platelet-neutrophil aggregates ( $n=6-9$ mice per group). (C) Platelet depletion by Abs prior to initiation of acid-induced ALI reduced the amount of platelet-neutrophil interactions in the blood. (D) Platelet-neutrophil interactions 30 minutes after initiation of acid-induced $\mathrm{ALI}$ in pulmonary microvasculature visualized by electron microscopy. Platelets (arrows) attached directly to the endothelium and a neutrophil. Scale bar: $1 \mu \mathrm{m}$. Original magnification, $\times 8000 .{ }^{\#} P<0.05$. P-sel, P-selectin. tion of intravascular (Figure 3E) and interstitial PMN accumulation (data not shown) and permeability (Figure 3G) in response to $\mathrm{HCl}$ instillation. When WT mice were reconstituted with $\mathrm{BM}$ from selectin $P^{-/-}\left(\right.$Selp $\left.^{-/-}\right)$mice, gas exchange was significantly improved (Figure 3D), and intravascular (Figure 3E), interstitial (data not shown), and intraalveolar PMN accumulation (Figure 3F) as well as permeability (Figure $3 \mathrm{G}$ ) were reduced. When Selp $\mathrm{S}^{-/}$mice were reconstituted with BM from WT mice, intravascular PMN accumulation was reduced, but all other functional and morphological parameters were similar to those seen in WT mice. These results show that platelet and not endothelial P-selectin is responsible for the pathology of acid-induced ALI.

Neutrophil-platelet aggregates interact with endothelial cells. In order to elucidate the molecular mechanisms responsible for our in vivo results, we investigated the role of different cell populations and activation states in PMN adhesion to human pulmonary microvascular endothelial cells (HPMECs). Incubation of activated PMNs or the combination of unstimulated PMNs and platelets induced a small increase in PMN adhesion. The combination of activated PMNs with activated or resting platelets or resting PMNs with activated platelets induced a further 3 -fold increase $(P<0.05)$ of PMN adhesion to endothelial cells (Figure 4A).

Platelet-neutrophil interaction is known to promote the production of $\mathrm{TXA}_{2}$ (32). To differentiate between the importance of endothelial and platelet TPs, we incubated endothelia with a specific TP antagonist for 2 hours. Blocking of endothelial TPs reduced PMN adhesion (Figure 4B). These data suggest that the interaction of platelets and PMNs augments adhesion of PMNs to endothelial cells by $\mathrm{TXA}_{2}$ effects on the endothelia.

A previous study demonstrated that acid-induced ALI is partially ICAM-1 dependent (33). We therefore studied whether plateletneutrophil interactions induced endothelial ICAM-1 expression.
We incubated PMNs, platelets, or both with HPMECs and determined endothelial ICAM-1 expression mRNA by quantitative realtime RT-PCR. Incubation of activated PMNs, platelets, or both resulted in a significant induction of endothelial ICAM-1 mRNA expression (Figure 4C). This was further enhanced when activated or resting platelets were added. Activated platelets together with resting PMNs induced as much endothelial ICAM-1 expression as did activated PMNs (Figure 4C). In order to show that the $\mathrm{TXA}_{2}$ release by platelet-neutrophil aggregates is responsible for the endothelial ICAM-1 mRNA expression, we incubated endothelia with a specific TP antagonist before we added the cells. The TP antagonist blocked the ICAM-1 mRNA expression by approximately $40 \%$ (data not shown).

To determine whether platelet interaction with neutrophils was sufficient to induce $\mathrm{TXA}_{2}$ synthesis, isolated human platelets were incubated with isolated human neutrophils for 2 hours at $37^{\circ} \mathrm{C}$. This resulted in a 2.5 -fold increase in $\mathrm{TXB}_{2}$ measured in the supernatant, which was further enhanced to 5.5-fold when either PMNs or platelets were activated by TNF- $\alpha$ or thrombin, respectively (Figure 4D).

Blocking $T X A_{2}$ prevents acid-induced ALI. In order to test the biological significance of our in vitro findings in vivo, we measured $\mathrm{TXB}_{2}$, a stable degradation product of $\mathrm{TXA}_{2}$, in mouse plasma under baseline conditions and after platelet depletion. Two hours after intratracheal $\mathrm{HCl}$ application, $\mathrm{TXB}_{2}$ concentration in the plasma increased significantly, consistent with a previous study (7). The depletion of platelets by busulfan or Abs prior to induction of ALI significantly decreased plasma $\mathrm{TXB}_{2}$ (Figure $5 \mathrm{~A}$ and data not shown).

To determine whether TXA 2 is an important mediator of ALI, we pretreated acid-exposed mice with a specific TP antagonist. This improved gas exchange (Figure 5B) and reduced intravascular, interstitial (data not shown), and intraalveolar (Figure 5C) PMN 
A

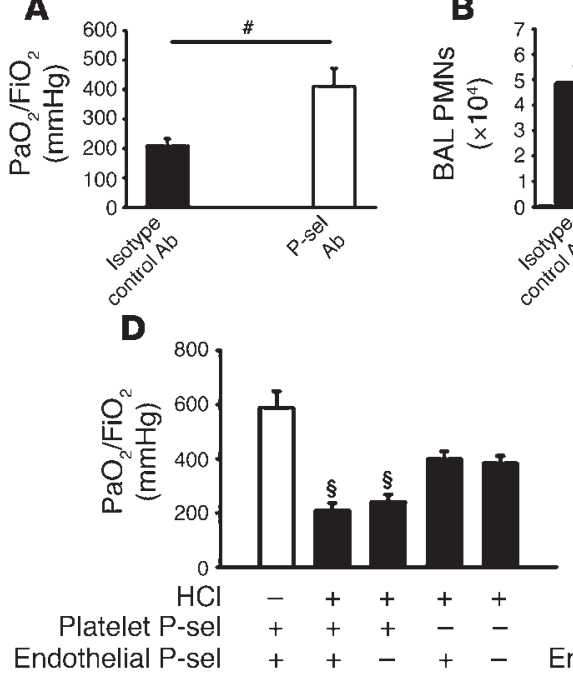

Endothelial P-sel ++-++

$\mathbf{F}$

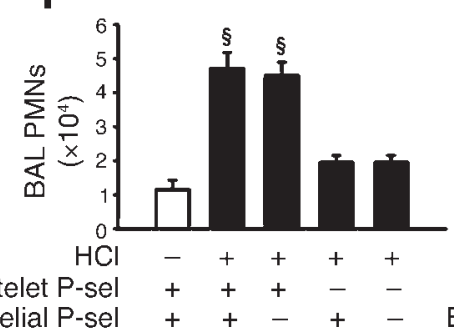

$\because$

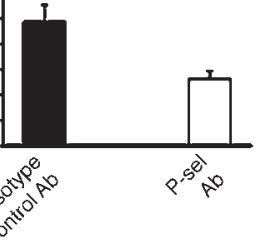

E

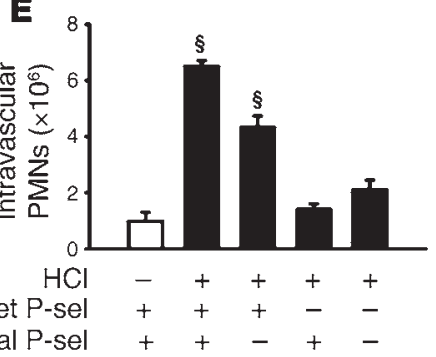

G

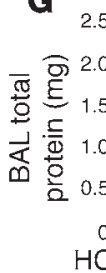

Platelet P-sel Endothelial P-sel

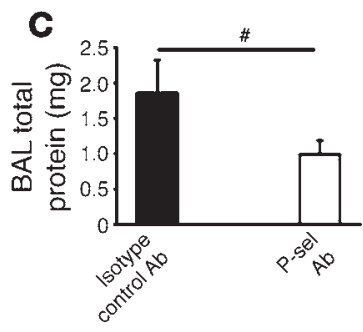

Figure 3

Platelet P-selectin plays key role in the development of ALI. (A-C) Injecting $P$-selectin Abs 15 minutes after the induction of ALI ( $n=4-5$ mice per group) improved gas exchange (A) and reduced interstitial (data not shown) and BAL fluid (B) PMNs as well as permeability (C). (D-G) To determine whether hematopoietic (platelet) or nonhematopoietic (endothelial) P-selectin is responsible for neutrophil recruitment in ALI, BM chimeras (WT into WT; WT into Selp $p^{-/-}$; Selp $p^{-/-}$into WT; Selp $p^{-/-}$into Selp $\left.p^{--}\right)$were tested $(n=4-5$ mice per group). Mice lacking platelet P-selectin showed improved gas exchange (D), reduced $\mathrm{PMN}$ accumulation in the intravascular (E), interstitial (data not shown), and alveolar compartments $(\mathbf{F})$, and diminished permeability (G) compared with mice expressing hematopoietic P-selectin. ${ }^{\#} P<0.05 ; \$ P<0.05$ versus platelet Selp ${ }^{-1-}$. accumulation $(P<0.05)$. TP blockade almost completely normalized permeability (Figure 5D), suggesting that $\mathrm{TXA}_{2}$ is the main mediator of permeability in this model of ALI. Pretreatment with the TP antagonist before induction of ALI reduced alveolar septal thickening, accumulation of cells within the interstitial compartment, and influx of protein-rich fluid into the alveolar space in the histological analysis of the lungs (Supplemental Figure 1B). Acetylsalicylic acid (ASS) at a dose that completely blocks cyclooxygenase and thus thromboxane synthesis (30) had similar effects, but was not as effective (Figure 5, B-D) as blocking TPs.

$\mathrm{TX}_{2}$ and neutrophil-platelet interactions induce endothelial cell contraction and determine disease model outcome. To show direct effects of TXA on endothelial cells, we measured cytoskeletal F-actin in HPMECs under different conditions. TP stimulation with a $\mathrm{TXA}_{2}$ analogue induced a striking increase in F-actin polymerization. F-actin rings appeared mainly at the edge of the cells and caused cell retraction. These changes were dose and time dependent (Figure 6A). To determine the cellular source of $\mathrm{TXA}_{2}$, we used a coculture system in which HPMECs were incubated with freshly isolated human PMNs and/or platelets. F-actin formation was quantified by measuring released FITC-phalloidin in the supernatant. Activated PMNs increased F-actin formation, which was similar to the effect seen by adding PMNs and platelets, when at least 1 cell type was activated. The combination of activated platelets and activated PMNs induced a further increase of F-actin polymerization (Figure 6B).

To determine the importance of platelet depletion, TP blockade, and P-selectin blockade in vivo, we investigated the disease model outcome. Acid-induced ALI led to the death of all mice within 3 hours (Figure 6C) whereas all saline-treated control mice survived (data not shown). Treatment of acid-treated mice with TP antagonist or platelet depletion by busulfan prior to induction of ALI caused a prolongation of survival. Remarkably, P-selectin Abs given as a therapeutic approach 15 minutes after induction of ALI led to survival of all mice until the end of the study (Figure 6C).

Platelets influence sepsis-induced ALI. In order to address the question of whether platelet-neutrophil interactions are also involved in development of ALI caused by sepsis, we investigated the role of this cell interaction in a zymosan/LPS-induced ALI model. Four hours after induction of ALI, mice showed significantly reduced gas exchange (Figure 7A), increased accumulation of neutrophils in the intravascular (Figure 7B) and alveolar (Figure 7C) compartments, and permeability (Figure 7D). Platelet depletion by Abs prior to induction of the sepsis model caused ameliorated gas exchange, reduced permeability (Figure 7D), and neutrophil accumulation in the intravascular (Figure 7B) and alveolar (Figure 7C) spaces.

\section{Discussion}

The results of our study suggest that platelet-neutrophil interactions play a crucial role in the development of acid-induced ALI. Our model is severe and lethal, as opposed to a milder injury in mice ventilated with a high concentration of inspiratory oxygen (6). Depletion of platelets or disruption of platelet-neutrophil interactions reduced PMN migration and permeability and improved gas exchange after acid application, resulting in prolonged survival. Increased neutrophil adhesion and activation of endothelia was caused by TXA 2 produced by platelet-neutrophil aggregates. These observations indicate that platelet-neutrophil interactions are critical to the development of ALI, to which they contribute by enhancing their respective activations, which trigger the production of $\mathrm{TXA}_{2}$ and other inflammatory mediators. 


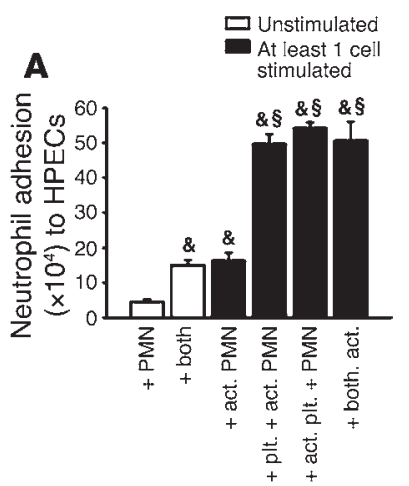

C

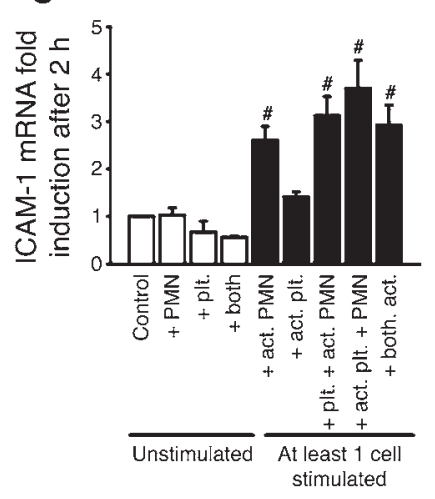

D
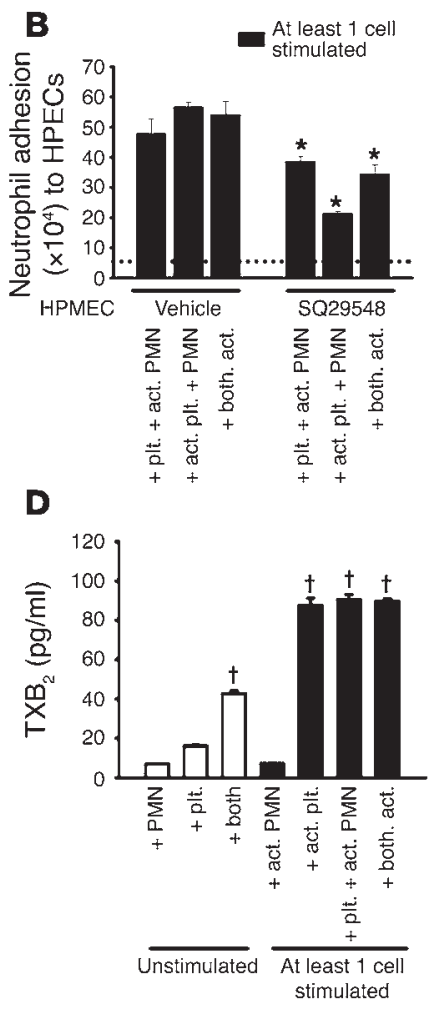

Pulmonary aspiration of gastric content induces ALI in 26\%-36\% of all cases and is associated with a high mortality $(34,35)$. Aspiration of acid may damage the alveolar-capillary membrane and induce a release of inflammatory mediators, inflammatory cells,

\section{Figure 4}

Neutrophil-platelet adhesion to untreated HPMECs requires endothelial cell TPs. (A) Platelet-neutrophil interactions significantly induced PMN adhesion ( $n=3$ per group). (B) Increased adhesion was partially mediated by endothelial cell TPs. Baseline PMN adhesion is indicated by dotted line. (C) ICAM-1 mRNA in endothelial cells was increased by exposure to activated PMNs or the combination of PMNs and platelets when at least 1 of the cell types was activated ( $n=3$ per group). (D) The incubation of human platelets with neutrophils induced a 2.5fold increase in $\mathrm{TXB}_{2}$ measured in the supernatant, which was further enhanced to 5.5 -fold when either PMNs or platelets were activated by TNF- $\alpha$ or thrombin. $n=3$ per group. $\& P<0.05$ versus PMNs; $\$ P<0.05$ versus both and activated PMNs; ${ }^{*} P<0.05$ versus vehicle; $\#<0.05$ versus control; ${ }^{\dagger} P<0.05$ versus platelets. Act., activated; plt., platelet.

expression of adhesion molecules, and enzymes, including TNF- $\alpha$, IL-8, cyclooxygenase and lipoxygenase products, and reactive oxygen species (4). Furthermore, these inflammatory processes may also lead to a coagulopathy, which results from activation of coagulation and inhibition of fibrinolysis (36). Here we show that preventing platelet-neutrophil interactions can completely reverse this process. First, platelets control neutrophil recruitment into the lung in this and a second, sepsis-induced model. Second, induction of ALI leads to a significant increase of platelet-neutrophil aggregates. Third, blocking P-selectin by Abs after induction of ALI as a therapeutic approach reduces formation of platelet-neutrophil aggregates and development of ALI and leads to prolonged survival. Platelet but not endothelial P-selectin is responsible for the development of ALI.

Our in vitro studies show that activation of either PMNs or platelets alone has the same effects as simultaneous activation of both cell types, suggesting that PMNs and platelets activate each other. This interpretation is consistent with a previous study in a

\section{Figure 5}

Blocking $\mathrm{TXA}_{2}$ prevents acid-induced ALI. (A) ALI induced increased plasma $\mathrm{TXB}_{2}$ levels at 2 hours. Platelet depletion partially reduced $\mathrm{TXB}_{2}$ levels $(n=4-5$ mice per group). (B-D) Blocking of TPs ( $n=4-5$ mice per group) significantly improved gas exchange (B), reduced PMN accumulation in the intravascular, interstitial (data not shown), and alveolar compartments (C), and prevented increased vascular permeability (D). ASS showed similar effects regarding PMN recruitment and permeability (C-D), but the effect on gas exchange was not as pronounced as in TP blockade (B). ${ }^{\#} P<0.05$.
A
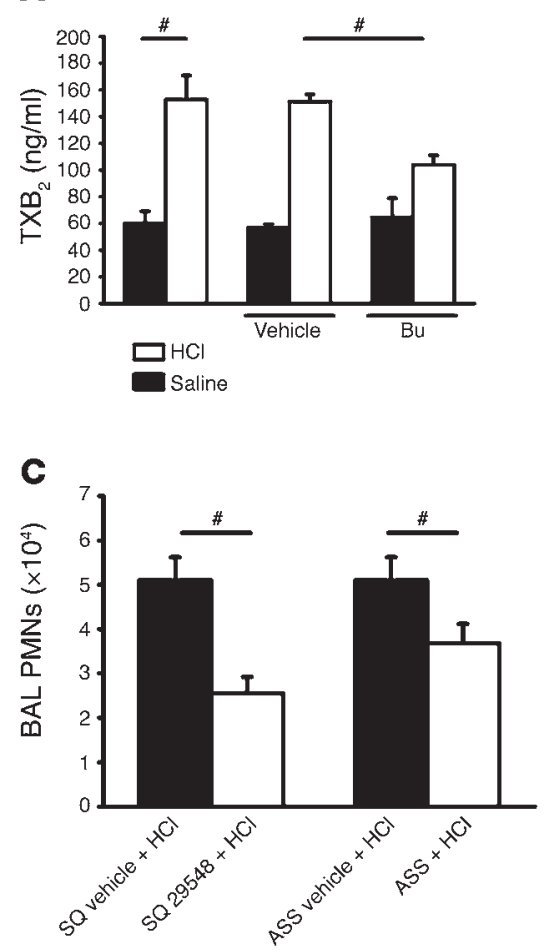
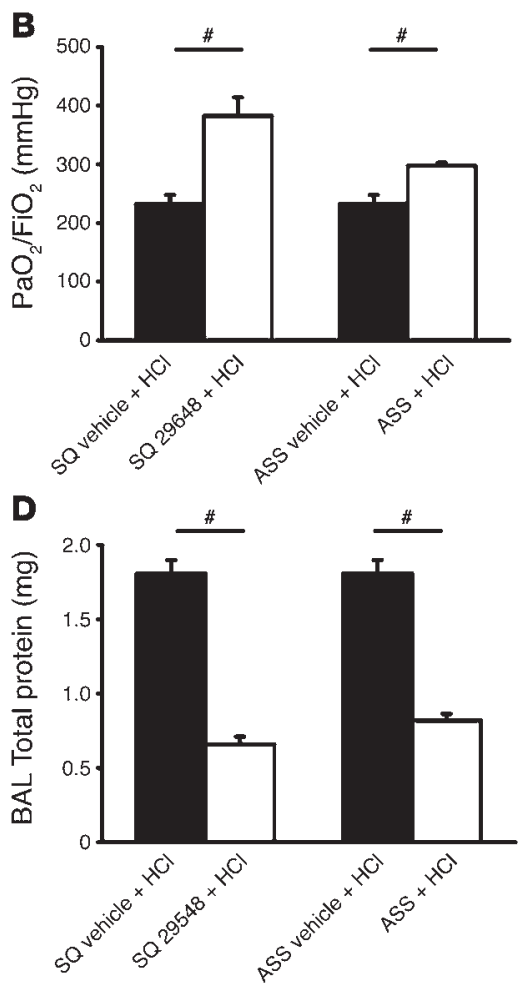
A
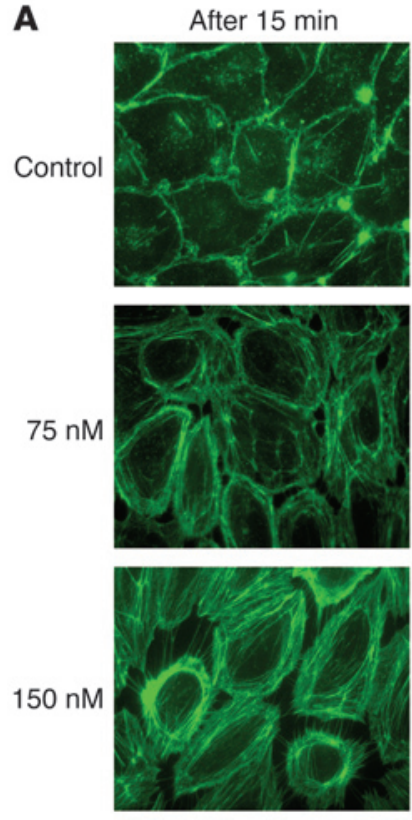

After $30 \mathrm{~min}$
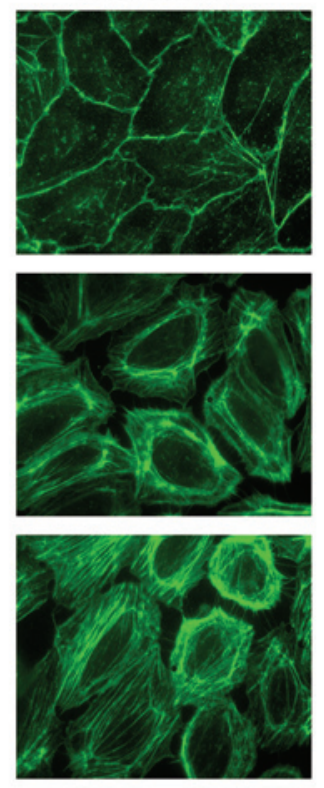

B

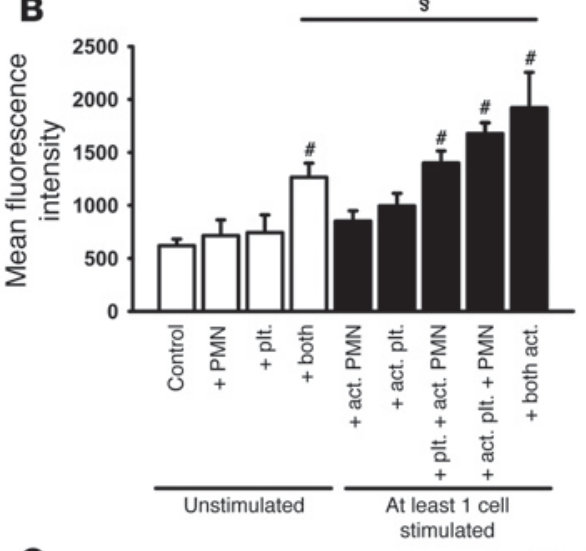

C

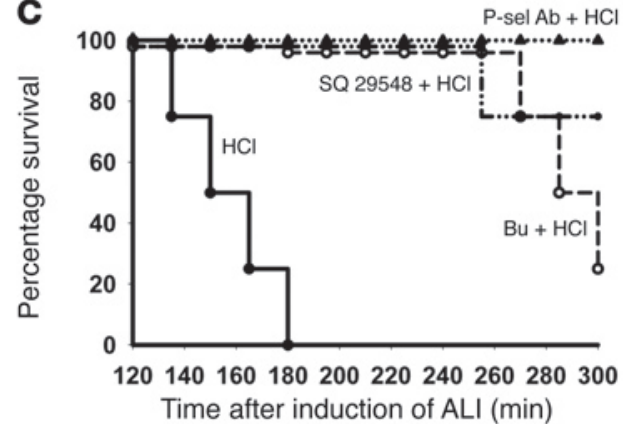

Figure 6

Endothelial cell response to TP activation as reflected by F-actin localization and content. (A) Human pulmonary endothelial cells were treated with 75 or $150 \mathrm{nM}$ of $\mathrm{TXA}_{2}$ analogue (SQ 29548), and F-actin was localized by phalloidin staining. Images are representative of 3 experiments with similar results. Original magnification, $\times 175$. (B) Activated platelets and activated PMNs induced a significant increase of F-actin formation in endothelial cells ( $n=3$ per group). The combination of stimulated platelets and PMNs caused a further increase of F-actin polymerization. $\$ P<0.05$; $\# P<0.05$ versus control. (C) Platelet depletion with busulfan or TP antagonist SQ 29548 prior to induction of ALI led to significant prolongation of survival compared with that of $\mathrm{HCl}$-treated mice ( $n=4$ per group). All mice treated with a P-selectin Ab survived until termination of the experiment ( 300 min), as did control mice (data not shown). The $\mathrm{HCl}$ group was significantly different from the other groups. $P=0.0002$ by log rank test.

different disease model showing that platelets present proinflammatory mediators on their surface to leukocytes and endothelia upon activation (37). When the interaction of platelets with endothelial cells or leukocytes is disturbed, mediator production and presentation are reduced.

During the interaction of activated platelets with leukocytes and endothelial cells, the platelets deposit platelet-derived mediators, including the chemokines CXCL4, CXCL7, and CXCL8. These mediators induce PMN activation, chemotaxis, adhesion, degranulation of primary and secondary granules, and production of reactive oxygen species (38-40). $\mathrm{TXA}_{2}$, an important lipid mediator of platelets, is involved in platelet-neutrophil interactions (41) and plays a critical role in acid-induced $\operatorname{ALI}(7,42)$. $\mathrm{TXA}_{2}$ induces increased permeability (42) and PMN adhesion to pulmonary endothelial cells (43). TP blocking or thromboxane synthesis inhibition was previously shown to protect from acidinduced PMN sequestration and protein leakage $(42,44)$. In our in vivo study, we demonstrate that platelets are a main source of $\mathrm{TXA}_{2}$ upon intratracheal acid application. Our in vitro studies show that the interaction of platelets with PMNs increases F-actin polymerization in endothelial cells and augments adhesion of PMNs to endothelial cells by TXA 2 effects on the endothelia.

Currently, there are no drugs to effectively manage pulmonary edema and PMN sequestration and to improve survival rates in
ALI. The present data suggest that blocking TPs or P-selectin represents potential therapeutic approaches to controlling acidinduced ALI. Both TP antagonists and P-selectin blocking drugs are under development. Our data show that the cyclooxygenase inhibitor ASS significantly improved oxygenation and reduced neutrophil recruitment and edema in ALI. However, this treatment was less effective than blocking TPs. ASS would be expected to reduce biosynthesis of all prostanoids, including antiinflammatory arachidonic acid derivatives such as prostacyclin, whereas $\mathrm{TP}$ inhibitors would selectively block the action of $\mathrm{TXA}_{2}$. A recent clinical trial using ketoconazole showed no benefit (45). This study included patients with different causes of directly (pneumonia, aspiration of gastric contents) and indirectly (e.g., sepsis, transfusion) induced ALI. Importantly, the measured $\mathrm{TXB}_{2}$ concentration in patients treated with ketoconazole was not reduced compared with the control group. Furthermore, the drug was administered a long time after the onset of ALI.

Our data provide what we believe is the first direct evidence for a contribution of circulating platelets in the development of acidand sepsis-induced ALI. P-selectin-dependent platelet-neutrophil interactions induced presentation of platelet-derived proinflammatory mediators to the endothelia and neutrophils, resulting in increased neutrophil adhesion and recruitment, activation of endothelial cells, and development of ALI. Therapeutic inhibition 
A
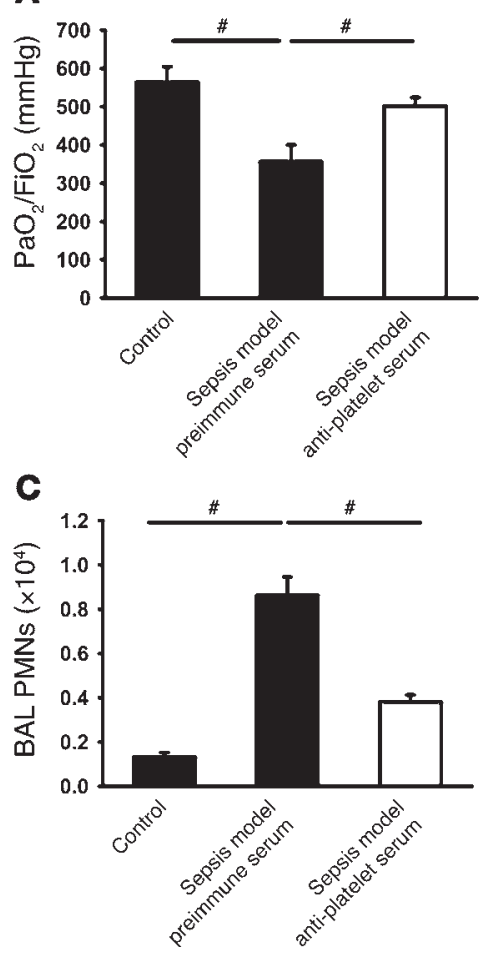

B
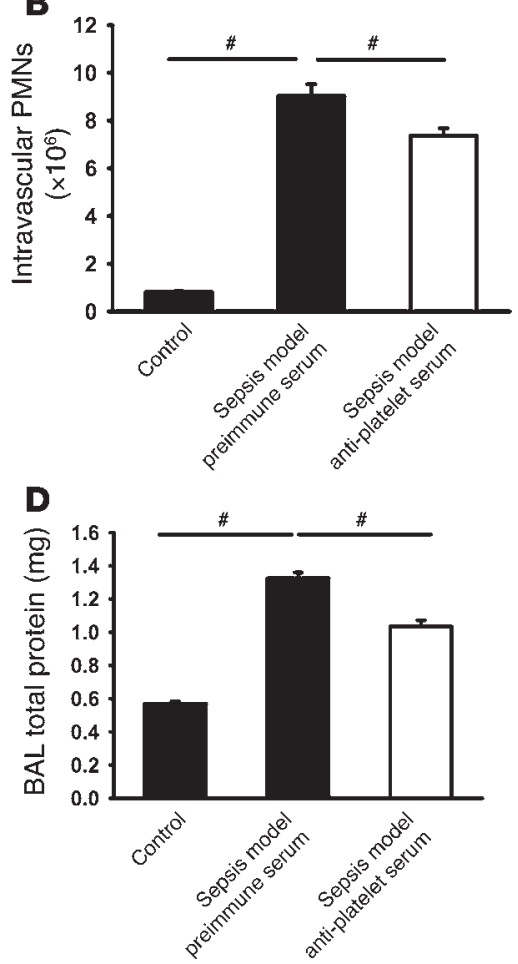

\section{Figure 7}

Platelets influence sepsis-induced ALI. (A-D) Four hours after initiation of sepsis-induced ALI by LPS and zymosan, mice displayed reduced gas exchange (A), increased accumulation of neutrophils in the intravascu$\operatorname{lar}(\mathbf{B})$ and alveolar (C) compartments, and permeability (D). Platelet depletion by Ab prior to the induction of the sepsis model caused improved gas exchange, reduced permeability (D), and neutrophil accumulation in the intravascular (B) and alveolar (C) spaces. ${ }^{\#} P<0.05$. of platelet neutrophil aggregates and/or neutralization of platelet-derived proinflammatory mediators may provide a novel therapeutic approach to ALI that should be explored in clinical trials.

\section{Methods}

Animals. We used 8- to 12-week-old C57BL/6 mice (The Jackson Laboratory) and P-selectin-deficient mice (C57BL/6 for at least 10 generations). Mice were housed in a barrier facility under specific pathogen-free conditions. The Animal Care and Use Committee of the University of Virginia approved all animal experiments.

Acid-induced ALI. Mice were anesthetized with i.p. injections of ketamine (125 $\mu \mathrm{g} / \mathrm{g}$ body weight; Sanofi-Aventis), xylazine (12.5 $\mu \mathrm{g} / \mathrm{g}$ body weight; Phoenix Scientific Inc.), and atropine sulfate $(0.025 \mu \mathrm{g} / \mathrm{g}$ body weight; Fujisawa) and were placed on a heating pad to maintain body temperature. In order to effect acid-induced ALI, mice received $2 \mu \mathrm{l} / \mathrm{g}$ of $1 \mathrm{M} \mathrm{HCl}(\mathrm{pH}=1.5)$ intratracheally, followed by a bolus of air $(30 \mu \mathrm{l} / \mathrm{g})$. Following a tracheotomy, mice were ventilated with a respirator (MiniVent, Type 845; Hugo Sachs Elektronik) for 2 hours (tidal volume, $10 \mu \mathrm{l} / \mathrm{g}$; respiration rate, 140/min; fraction of inspiratory oxygen $\left.\left[\mathrm{FiO}_{2}\right], 0.21\right)$. Control animals received saline instead of $\mathrm{HCl}$ in the same manner.

Sepsis-induced ALI. ALI was induced as previously described with some modifications (7). In brief, mice were anesthetized and mechanically ventilated. One minute prior to induction of ALI, 3 deep inhalations $(3 \times$ tidal volume) were applied. Subsequently, mice were injected with $3 \mu \mathrm{g} / \mathrm{g}$ of LPS i.v. (Escherichia coli O111:B4; Sigma-Aldrich), and 2 hours later, they received $7 \mu \mathrm{g} / \mathrm{g}$ zymosan A (Saccharomyces cerevisiae; Sigma-Aldrich) i.v. The control group received saline instead of LPS and zymosan A in the same manner. The experiment was terminated 4 hours after induction of ALI.

Platelet depletion. In order to investigate the role of platelets in the recruitment of PMNs into the lung and the development of ALI, platelets were depleted as previously described (46). Briefly, mice received 2 injections of busulfan $(20 \mathrm{mg} / \mathrm{kg}$ dissolved in polyethylene glycol 400) or vehicle (poly- ethylene glycol 400) on days 0 and 3. Experiments were performed on day 14. Immediately before experiments, blood counts were determined (HEMAVET 850 FS; CDC Technologies Inc.). Platelet depletion by busulfan led to a significant reduction of platelets without affecting leukocytes (Supplemental Table 1A). In another set of experiments, we used a rabbit anti-mouse platelet serum (Accurate) to deplete platelets. Two hours prior to induction of ALI, mice were injected i.p. with $25 \mu \mathrm{l}$ of the Abs. This method led to a significant reduction (85\%) of platelets in the systemic circulation without affecting leukocytes (data not shown). Control mice received preimmune serum.

$P M N$ recruitment into the lung. After euthanasia, BAL fluid was collected $(5 \times 1 \mathrm{ml}$ phosphate-buffered saline). After BAL fluid was centrifuged, PMNs in the BAL fluid were counted using Kimura stain. The protein concentration of the supernatant was determined by the Lowry method.

In order to distinguish between the localization of intravascular and interstitial PMNs, we used a recently developed flow cytometry-based method to determine pulmonary PMN extravasation (11). In brief, Alexa Fluor 633labeled Gr-1 Abs (clone RB6-8C5, purified from the supernatant of the Gr-1 hybridoma [ATCC] at the biomolecular facility of the University of Virginia; Alexa Fluor 633 Protein Labeling Kit [Invitrogen]) to murine PMNs, which label all intravascular but not interstitial or alveolar PMNs (11), were injected i.v. 5 minutes prior to anesthetic overdose. After collecting BAL fluid, the inferior vena cava was opened and nonadherent PMNs were dislodged from the pulmonary vasculature by flushing $10 \mathrm{ml}$ of PBS at $25 \mathrm{~cm} \mathrm{H}_{2} \mathrm{O}$ through the right ventricle. Lungs were removed and minced, and the samples were incubated with $125 \mathrm{U} / \mathrm{ml}$ collagenase type XI, $60 \mathrm{U} / \mathrm{ml}$ hyaluronidase type I-s, $60 \mathrm{U} / \mathrm{ml}$ DNase 1 , and unlabeled anti-Gr- 1 at $37^{\circ} \mathrm{C}$ for 60 minutes in order to prevent possible binding of the injected Abs to extravascular PMNs. A cell suspension was made by passing the digested lungs through a $70-\mu \mathrm{m}$ cell strainer (BD Biosciences - Falcon). Erythrocytes were lysed and remaining leukocytes were resuspended and counted. The fraction of PMNs in the suspension was determined by flow cytometry (FACSCalibur; BD). PMNs were identified by their typical appearance in the forward/side scatter profiles 
and their expression of CD45 (clone 30-F11; BD Biosciences - Pharmingen), 7/4 (clone 7/4; BD Biosciences - Pharmingen), and Gr-1 (clone RB6-8C5). Isotype controls were used to compensate for nonspecific $\mathrm{Ab}$ binding. The labeled $\mathrm{Gr}-1 \mathrm{Ab}$ was used to differentiate between intravascu$\operatorname{lar}\left(\mathrm{CD}^{+} 5^{+} 7 / 4^{+} \mathrm{Gr}-1^{+}\right)$and interstitial $\left(\mathrm{CD}^{+} 5^{+} 7 / 4^{+} \mathrm{Gr}-1^{-}\right)$PMNs.

Pulmonary function-oxygenation. Arterial blood was obtained from an arterial catheter, and standard arterial blood gas analyses were accomplished (Rapidlab 800 System; Bayer HealthCare). To normalize the partial pressure of arterial oxygen $\left(\mathrm{PaO}_{2}\right)$ to the fraction of inspiratory oxygen $\left(\mathrm{FiO}_{2}\right)$, the ratio of $\mathrm{PaO}_{2}$ to $\mathrm{FiO}_{2}\left(\mathrm{PaO}_{2} / \mathrm{FiO}_{2}, \mathrm{mmHg}\right.$, oxygenation index $)$ was calculated.

Neutrophil-platelet interactions. In order to determine the amount of platelet-neutrophil aggregates, $100 \mu \mathrm{l}$ acid-citrate dextrose (ACD) anticoagulated blood was added to a saturating concentration of anti-CD41 (FITC), anti-CD45 (PerCP), anti-Gr-1 (Alexa Fluor 633), and anti-CD11b (PE). After incubation for 10 minutes at room temperature, cells were fixed using paraformaldehyde. The number of neutrophil-platelet interactions were determined by flow cytometry (FACSCalibur; BD) within 4 hours of blood withdrawal.

Generation of P-selectin chimeric mice. To distinguish the role of nonhematopoietic and hematopoietic P-selectin for the development of ALI, chimeric mice were generated following a previously described protocol (47). C57BL/6 and P-selectin-deficient mice were used as donors and/or recipients. Recipient mice were lethally irradiated in 2 doses of 6 Gy each (separated by 4 hours). BM was isolated from female donor mice under sterile conditions, and approximately $5 \times 10^{6}$ cells were injected i.v. into male recipient mice. Experiments were performed 6 weeks after BM transplantation. To confirm complete reconstitution of the transferred BM, DNA of leukocytes was investigated for an area specific to the Y chromosome by real-time RT-PCR (primers: forward, 5 '-AGCAAACACCTCTACTACCCTCTA-3'; reverse, 5'-GGGCTGCATCAATTCAAATACCA-3'; probe, 5'-ACCTAAACGGTGGCCCCTCGGGG-3').

Effects of intervention. WT mice were used to asses the effects of P-selectin $\mathrm{mAb}(30 \mu \mathrm{g} / \mathrm{mouse}$, clone RB40.34), specific TP antagonist (SQ 29548, $2 \mathrm{mg} / \mathrm{kg}$, and $2 \mathrm{mg} / \mathrm{kg} / \mathrm{h}$; Cayman Chemical), or the cyclooxygenase inhibitor ASS (1 mg/g). SQ 29548 and ASS were injected 15 minutes prior to induction of ALI. P-selectin $\mathrm{mAb}$ was applied as a therapeutic approach 15 minutes after ALI induction.

Histology. In order to visualize morphological changes of acid-induced ALI, paraffin-embedded lung sections $(5 \mu \mathrm{m})$ were stained with H\&E. For transmission electron microscopy, tissue samples were fixed in glutaraldehyde (2.5\%) and paraformaldehyde (3.3\%) in phosphate buffer ( $\mathrm{pH}$ 7.2). After postfixation, ultrathin sections were cut with a diamond knife and ultramicrotome. The sections were collected on 200 mesh copper grids (Ernest F. Fullam Inc.), contrast stained with uranyl acetate (50\% in acetone) and lead citrate, and examined in a JEOL 100-CX transmission electron microscope.

Thromboxane measurement. Since $\mathrm{TXA}_{2}$ is rapidly degraded, $\mathrm{TXB}_{2}$ levels in plasma were determined by using immunoassay (EIA) kits (Amersham Biosciences).

Cell culture and platelet and neutrophil isolation and activation. HPMECs were maintained according to manufacturer's instructions (Cambrex) and used between passages 6 and 9. Human PMNs were isolated by Ficoll (48) whereas human platelets were isolated by gel filtration (37). For some experiments, PMNs were stained with calcein AM (Invitrogen). PMNs were activated with TNF- $\alpha(10 \mathrm{ng} / \mathrm{ml}$ for $15 \mathrm{~min})$ and platelets with thrombin $(0.1 \mathrm{U} / \mathrm{ml}$ for $10 \mathrm{~min})$, respectively. Monolayers of HPMECs were incubated with activated and/or inactivated cells for 30 minutes.

Assessment and quantification of F-actin in endothelial cells. To assess the distribution of F-actin upon $\mathrm{TXA}_{2}$-analogue stimulation (U46619; Cayman Chemical), HPMECs were cultured on glass coverslips. After stimulation, cells were fixed ( $4 \%$ formaldehyde), permeabilized (0.1\% Triton X-100), and stained with FITC-phalloidin (Invitrogen). Coverslips were mounted on glass slides, and microscopy was accomplished on a Nikon Diaphot inverted fluorescence microscope.

In separate experiments, platelets and/or PMNs were incubated with endothelial cells at $37^{\circ} \mathrm{C}$ for 30 minutes; PMNs and/or platelets were removed by washing. Cells were fixed, permeabilized, and stained as above. After the cells were washed, FITC-phalloidin was extracted from cells by incubation for 1 hour with $1 \mathrm{ml}$ methanol at $37^{\circ} \mathrm{C}$. Fluorescence of methanol supernatants was quantified on a plate reader.

$P M N-$ endothelial cell adhesion assay. Calcein AM-labeled cells $\left(1 \times 10^{5}\right.$ neutrophils and/or $1 \times 10^{6}$ platelets) were incubated with pulmonary endothelia for 30 minutes at $37^{\circ} \mathrm{C}$, nonadherent cells were removed by gentle washing and aspiration, and adherent PMNs were lysed and quantified in a fluorescence spectrometer. For blocking experiments, endothelial cells were incubated with specific TP antagonist (SQ 29548, $10 \mu \mathrm{M}$ ) for 2 hours. After TP blocking, antagonist was removed by washing.

Quantitative real-time RT-PCR. Two hours after incubation of endothelial cells with neutrophils and/or platelets, RNA was isolated using TRIzOL reagent (Invitrogen). Total RNA (900 ng) was reverse transcribed with murine leukemia virus (MuLV) reverse transcriptase using the GeneAmp RNA PCR kit (Applied Biosystems) and oligo dT primers. The mRNA sequences of the investigated genes were obtained from GenBank. The primers for $\beta_{2}$ microglobulin were described previously (49). The primers for ICAM-1 were designed using Primer3 software from the Whitehead Institute for Biomedical Research. The following primers were used: 5'-GGCTGGAGCTGTTTGAGAAC-3' (forward) and 5'-ACTGTGGGGTTCAACCTCTG-3' (reverse). Quantitative real-time RT-PCR was performed using iCycler iQ Real-Time Detection System technology (Bio-Rad). Quantification of target gene expression was performed using a mathematical model (50). The expression of the target molecule was normalized to the expression of $\beta_{2}$ microglobulin. To exclude contributions of ICAM-1 mRNA from neutrophils or platelets, these cell populations were isolated and RT-PCR was performed as mentioned above. Neither platelets nor PMNs expressed ICAM-1 mRNA (data not shown).

Statistics. Statistical analysis was performed with SPSS version 9.0 for Windows (SPSS) and included 1-way analysis of variance, Student-Newman-Keuls test, and 2-tailed Student's $t$ test where appropriate. KaplanMeier method was used for analyzing survival rates. All data are presented as mean \pm SEM. $P<0.05$ was considered significant.

\section{Acknowledgments}

The authors wish to thank Tracy Burcin for technical support, Emily Smith for critical reading, and Jan Redick, Advanced Microscopy Facility, University of Virginia, for her excellent technical assistance with transmission electron microscopy. This study was supported by the Deutsche Forschungsgemeinschaft (grant AZ 428/2-1 to A. Zarbock) and by an NIH grant (PO1 HL 73361, Project 2, to K. Ley).

Received for publication June 26, 2006, and accepted in revised form September 19, 2006.

Address correspondence to: Klaus Ley, University of Virginia Health System, Cardiovascular Research Center, PO Box 801394, Charlottesville, Virginia 22908-1394, USA. Phone: (434) 243-9966; Fax: (434) 924-2828; E-mail: klausley@virginia.edu.

Kai Singbartl's present address is: Department of Critical Care Medicine, University of Pittsburgh, Pittsburgh, Pennsylvania, USA. 
1. Rubenfeld, G.D., et al. 2005. Incidence and outcomes of acute lung injury. N. Engl. J. Med. 353:1685-1693.

2. Warner, M.A., Warner, M.E., and Weber, J.G. 1993. Clinical significance of pulmonary aspiration during the perioperative period. Anesthesiology. 78:56-62.

3. Olsson, G.L., Hallen, B., and Hambraeus-Jonzon, K. 1986. Aspiration during anaesthesia: a computeraided study of 185,358 anaesthetics. Acta Anaesthesiol. Scand. 30:84-92.

4. Marik, P.E. 2001. Aspiration pneumonitis and aspiration pneumonia. N. Engl. J. Med. 344:665-671.

5. Imai, Y., et al. 2003. Injurious mechanical ventilation and end-organ epithelial cell apoptosis and organ dysfunction in an experimental model of acute respiratory distress syndrome. JAMA. 289:2104-2112.

6. Imai, Y., et al. 2005. Angiotensin-converting enzyme 2 protects from severe acute lung failure. Nature. 436:112-116.

7. Nagase, T., et al. 2000. Acute lung injury by sepsis and acid aspiration: a key role for cytosolic phospholipase A2. Nat. Immunol. 1:42-46.

8. Eijking, E.P., Gommers, D., So, K.L., Vergeer, M., and Lachmann, B. 1993. Surfactant treatment of respiratory failure induced by hydrochloric acid aspiration in rats. Anesthesiology. 78:1145-1151.

9. Goldman, G., et al. 1992. Reactive oxygen species and elastase mediate lung permeability after acid aspiration. J. Appl. Physiol. 73:571-575.

10. Ware, L.B., and Matthay, M.A. 2000. The acute respiratory distress syndrome. N. Engl. J. Med. 342:1334-1349.

11. Reutershan, J., Basit, A., Galkina, E.V., and Ley, K. 2005. Sequential recruitment of neutrophils into lung and bronchoalveolar lavage fluid in LPSinduced acute lung injury. Am. J. Physiol. Lung Cell. Mol. Physiol. 289:L807-L815

12. Doerschuk, C.M. 2001. Mechanisms of leukocyte sequestration in inflamed lungs. Microcirculation. 8:71-88.

13. Kubes, P. 2002. The complexities of leukocyte recruitment. Semin. Immunol. 14:65-72.

14. Ostrovsky, L., et al. 1998. A juxtacrine mechanism for neutrophil adhesion on platelets involves platelet-activating factor and a selectin-dependent activation process. Blood. 91:3028-3036.

15. Romo, G.M., et al. 1999. The glycoprotein Ib-IX-V complex is a platelet counterreceptor for P-selectin. J. Exp. Med. 190:803-814.

16. Bombeli, T., Schwartz, B.R., and Harlan, J.M. 1998. Adhesion of activated platelets to endothelial cells: evidence for a GPIIbIIIa-dependent bridging mechanism and novel roles for endothelial intercellular adhesion molecule 1 (ICAM-1), alphavbeta 3 integrin, and GPIbalpha. J. Exp. Med. 187:329-339.

17. Frenette, P.S., et al. 1998. Platelet-endothelial interactions in inflamed mesenteric venules. Blood. 91:1318-1324.

18. Andre, P., et al. 2000. Platelets adhere to and translocate on von Willebrand factor presented by endothelium in stimulated veins. Blood. 96:3322-3328.
19. Pitchford, S.C., et al. 2005. Platelet P-selectin is required for pulmonary eosinophil and lymphocyte recruitment in a murine model of allergic inflammation. Blood. 105:2074-2081.

20. Carvalho-Tavares, J., et al. 2000. A role for platelets and endothelial selectins in tumor necrosis factoralpha-induced leukocyte recruitment in the brain microvasculature. Circ. Res. 87:1141-1148.

21. Hamburger, S.A., and McEver, R.P. 1990. GMP-140 mediates adhesion of stimulated platelets to neutrophils. Blood. 75:550-554.

22. Rinder, H.M., Bonan, J.L., Rinder, C.S., Ault, K.A., and Smith, B.R. 1991. Activated and unactivated platelet adhesion to monocytes and neutrophils. Blood. 78:1760-1769.

23. Blanks, J.E., Moll, T., Eytner, R., and Vestweber, D. 1998. Stimulation of P-selectin glycoprotein ligand-1 on mouse neutrophils activates beta 2 -integrin mediated cell attachment to ICAM-1. Eur. J. Immunol. 28:433-443.

24. Hidari, K.I., Weyrich, A.S., Zimmerman, G.A., and McEver, R.P. 1997. Engagement of P-selectin glycoprotein ligand-1 enhances tyrosine phosphorylation and activates mitogen-activated protein kinases in human neutrophils. J. Biol. Chem. 272:28750-28756.

25. Weyrich, A.S., McIntyre, T.M., McEver, R.P., Prescott, S.M., and Zimmerman, G.A. 1995. Monocyte tethering by P-selectin regulates monocyte chemotactic protein- 1 and tumor necrosis factor- $\alpha$ secretion. Signal integration and NF-K B translocation. J. Clin. Invest. 95:2297-2303.

26. Weber, C., and Springer, T.A. 1997. Neutrophil accumulation on activated, surface-adherent platelets in flow is mediated by interaction of Mac-1 with fibrinogen bound to $\alpha \operatorname{IIb} \beta 3$ and stimulated by platelet-activating factor. J. Clin. Invest. 100:2085-2093.

27. Zaitsu, M., et al. 2003. Thromboxane synthesis is increased by upregulation of cytosolic phospholipase A2 and cyclooxygenase- 2 in peripheral polymorphonuclear leukocytes during bacterial infection in childhood. Am. J. Hematol. 72:115-120.

28. Ruggeri, Z.M. 2002. Platelets in atherothrombosis. Nat. Med. 8:1227-1234.

29. Huang, J.S., Ramamurthy, S.K., Lin, X., and Le Breton, G.C. 2004. Cell signalling through thromboxane A2 receptors. Cell. Signal. 16:521-533.

30. Patrono, C., Garcia Rodriguez, L.A., Landolfi, R., and Baigent, C. 2005. Low-dose aspirin for the prevention of atherothrombosis. N. Engl. J. Med. 353:2373-2383.

31. Goff, C.D., et al. 1997. Postinjury thromboxane receptor blockade ameliorates acute lung injury. Ann. Thorac. Surg. 64:826-829.

32. Maugeri, N., et al. 1994. Polymorphonuclear leukocyte-platelet interaction: role of P-selectin in thromboxane B2 and leukotriene $\mathrm{C} 4$ cooperative synthesis. Thromb. Haemost. 72:450-456.

33. Nagase, T., et al. 1996. Intercellular adhesion molecule- 1 mediates acid aspiration-induced lung injury. Am. J. Respir. Crit. Care Med. 154:504-510.
34. Hudson, L.D., Milberg, J.A., Anardi, D., and Maunder, R.J. 1995. Clinical risks for development of the acute respiratory distress syndrome. Am. J. Respir. Crit. Care Med. 151:293-301.

35. Fowler, A.A., et al. 1983. Adult respiratory distress syndrome: risk with common predispositions. Ann. Intern. Med. 98:593-597.

36. Schultz, M.J., Haitsma, J.J., Zhang, H., and Slutsky, A.S. 2006. Pulmonary coagulopathy as a new target in therapeutic studies of acute lung injury or pneumonia-a review. Crit. Care Med. 34:871-877.

37. Huo, Y., et al. 2003. Circulating activated platelets exacerbate atherosclerosis in mice deficient in apolipoprotein E. Nat. Med. 9:61-67.

38. Brandt, E., Ludwig, A., Petersen, F., and Flad, H.D. 2000. Platelet-derived CXC chemokines: old players in new games. Immunol. Rev. 177:204-216.

39. Brandt, E., et al. 2000. The beta-thromboglobulins and platelet factor 4: blood platelet-derived CXC chemokines with divergent roles in early neutrophil regulation. J. Leukoc. Biol. 67:471-478.

40. Baggiolini, M., Dewald, B., and Moser, B. 1994. Interleukin-8 and related chemotactic cytokines-CXC and CC chemokines. Adv. Immunol. 55:97-179.

41. Chlopicki, S., Lomnicka, M., and Gryglewski, R.J. 2003. Obligatory role of lipid mediators in plateletneutrophil adhesion. Thromb. Res. 110:287-292.

42. Goldman, G., et al. 1991. Neutrophil accumulations due to pulmonary thromboxane synthesis mediate acid aspiration injury. J. Appl. Physiol. 70:1511-1517.

43. Wiles, M.E., Welbourn, R., Goldman, G., Hechtman, H.B., and Shepro, D. 1991. Thromboxane-induced neutrophil adhesion to pulmonary microvascular and aortic endothelium is regulated by CD18. Inflammation. 15:181-199.

44. Goldman, G., et al. 1992. Synergism between leukotriene B4 and thromboxane A2 in mediating acid-aspiration injury. Surgery. 111:55-61.

45. ARDS Network Authors. 2000. Ketoconazole for early treatment of acute lung injury and acute respiratory distress syndrome: a randomized controlled trial. The ARDS Network. JAMA. 283:1995-2002.

46. Kawasaki, T., et al. 2001. Mouse carotid artery ligation induces platelet-leukocyte-dependent luminal fibrin, required for neointima development. Circ. Res. 88:159-166.

47. Forlow, S.B., et al. 2001. Increased granulopoiesis through interleukin-17 and granulocyte colonystimulating factor in leukocyte adhesion moleculedeficient mice. Blood. 98:3309-3314.

48. Gamberale, R., Giordano, M., Trevani, A.S., Andonegui, G., and Geffner,J.R. 1998. Modulation of human neutrophil apoptosis by immune complexes. J. Immunol. 161:3666-3674.

49. Wellmann, S., et al. 2001. Specific reverse transcription-PCR quantification of vascular endothelial growth factor (VEGF) splice variants by LightCycler technology. Clin. Chem. 47:654-660.

50. Pfaffl, M.W. 2001. A new mathematical model for relative quantification in real-time RT-PCR. Nucleic Acids Res. 29:e45. 